\title{
Using a proforma to improve standards of documentation of an orthopaedic post- take ward round
}

Oliver Duxbury, Stephanie Hili, John Afolayan

\begin{abstract}
In Trauma and Orthopaedics, a daily Trauma Meeting (TM) occurs, where the previous 24-hour take is discussed and a management plan is decided by the consultant on-take. The post-take ward-round (PTWR) usually follows. In the district general hospital (DGH) where this audit was conducted, clinical incidents and root-cause analysis revealed that the TM/PTWR documentation were suboptimal.
\end{abstract}

We identified gold standards. Variables included clear documentation of PTWR, date/time, consultant on-take, clinician leading the wardround, and management plan. 50 cases were reviewed retrospectively.

72\% were seen on PTWR. 47\% of these were clearly labeled PTWR. $64 \%$ of the cases not seen on PTWR were weekend admissions. Documentation of the previously mentioned fields were also poor.

Audit results were presented at the department meeting and a Trauma Meeting/Post-Take Ward Round Proforma was implemented. A prospective re-audit of 50 cases revealed that patients not seen on a PTWR decreased to $18 \% ; 4 \%$ of these were weekend admissions. $88 \%$ of patients seen had a proforma completed. $18 \%$ of all cases did not have a proforma.

Introduction of the proforma established a system to document discussions at the TM and improved the quality of documentation of the consultant-led plans. Such a simple tool can improve the overall care of patients and potentially protect staff.

\section{Problem}

This audit was carried out at a DGH serving a population of 320,000 for general services with a 24-hour, seven days a week consultantled trauma service. A daily Trauma Meeting (TM) occurs during which the previous 24-hour take is discussed, relevant imaging reviewed and a management plan is decided by the consultant ontake. The post-take ward round (PTWR) usually follows this trauma meeting.

Junior doctors are relied upon to document the discussions of the TM and the PTWR. Previously there was no set system to document these discussions and the interaction with the patient. There were clinical incidences related to patient ownership and handing-over of care. After a systematic evaluation it was apparent that the PTWR documentation were suboptimal in these cases.

\section{Background}

Post take ward rounds can be high pace and there is a lot of pressure on junior doctors to document discussions and plans made by the clinician leading the ward round. It is vital that documentation of the PTWR and management plan is clear and readily available to all members of staff to help ensure the correct treatment plan is implemented efficiently with minimal chances of clinical incidences.
Many hospitals now use a proforma to enable clear, yet concise documentation of post take ward rounds. They enable other members of the multi disciplinary team to gain an understanding of the rational behind admission, the treatment plan for the patient and to identify the team who are responsible for the patient.

\section{Baseline measurement}

We identified gold standards using the GMC and the Royal College of Physicians recommendations (1,2). 50 trauma cases were selected randomly and the hospital notes were reviewed retrospectively. Audited variables included; presence of a clearly documented PTWR, date and time of admission, consultant ontake, clinician leading the ward-round and documenting, a clear documentation of the patientâcDs presenting compliant and a management plan.

$72 \%$ were seen on a PTWR and $47 \%$ of these had clearly labeled PTWR. $64 \%$ of the cases, which were not seen on a PTWR, had been admitted during the weekend. In $19 \%$ of cases there was no identifiable consultant on take, in $78 \%$ of cases the presenting complaint was not documented and there was no clear plan in $3 \%$. The entries were not dated in $3 \%$ of cases and not timed in $17 \%$ of cases. Details of the person documenting were also poor with $19 \%$ of case having no name of person documenting, $14 \%$ no signature; $50 \%$ no grade and $25 \%$ no contact details. 


\section{Design}

From the initial audit, it was clear that documentation was not acceptable. We therefore developed a Trauma Meeting/Post-Take Ward Round Proforma consisting of template for documentation of TM/PTWR including: patient details, date of admission, consultant and registrar on call, presenting complaint, management plan, details of the person documenting, and date and time.

\section{Strategy}

PDSA Cycle 1

The design of the proforma was discussed with Consultants, Registrars and Junior Doctors in orthopaedics, along with the trauma co-ordinator and other members of the multidisciplinary team who would be referring to the proforma on the wards.

\section{PDSA Cycle 2}

The results of the initial audit and the proforma were presented at the monthly Departmental Educational Half Day. Discussions from the meeting were positive but it was suggested that the proforma was on yellow paper, so that it would stand out in the notes.

\section{PDSA Cycle 3}

All surgical junior doctors who cover orthopaedics during weekends were informed of the new proforma and the need to attend trauma meeting when on call and to complete the profomas for the trauma admissions. Subsequently, the proforma was implemented and a second prospective audit was carried out 2 weeks later.

\section{Results}

A prospective re-audit was conducted two weeks after the intervention. 50 cases were reviewed whilst observing the same variables and the presence or absence of a TM/PTWR proforma.

The number of patients not seen on a PTWR decreased from $28 \%$ to $18 \% ; 4 \%$ of these were weekend admissions. $88 \%$ of patients seen on a PTWR had a proforma completed. $18 \%$ of all cases did not have a proforma. $67 \%$ of these were weekend admissions. In $4 \%$ of cases it was not possible to identify the responsible consultant. None of these had a proforma completed. In addition, $44 \%$ of the patients who were not seen on a PTWR had a clear consultant-formulated plan documented on the Trauma Meeting section of the proforma.

Of those cases seen on a PTWR, $2 \%$ did not have a clearly documented plan and $10 \%$ did not have the presenting complaint documented. In none of these cases was a proforma used to document the PTWR. All PTWR entries had been signed and dated. $12 \%$ did not have a name, $15 \%$ had no contact details and $10 \%$ had no grade. None of those without a name or grade had a PTWR proforma.
The introduction of the PTWR proforma when used greatly improved the level and quality of documentation of the post-take ward round. The introduction of a Trauma Meeting proforma not only established a system to document the discussion occurring at the meeting, but also meant that when patients were not seen on a PTWR, there was at least a clear documentation of management plan decided by a consultant. Completion of the proformas during weekends still needs to be improved.

\section{Conclusion}

The post-take surgical ward round is usually a very busy time for the junior doctors. Clear documentation in the notes serves as an important communication tool to the multiduciplinary team, it improves continuity with the handover system and it has increasing legal significance. A simple too such as a proforma and staff awareness can improve the overall care of patients and potentially protect staff.

\section{References}

1. Effective Approaches in Urgent and Emergency Care Paper 1 Priorities within Acute Hospitals. NHS Interim Management and Support (IMAS) November 2011.

2. "Good Clinical Care" Royal College of surgeons. http://www.rcseng.ac.uk/surgeons/working/professionalstandards/good-sur...

\section{Declaration of interests}

Nothing to declare

\section{Acknowledgements}

Fran Hole

\section{Lessons and limitations}

